PDES, SUBMANIFOLDS AND

AFFINE DIFFERENTIAL GEOMETRY

BANACH CENTER PUBLICATIONS, VOLUME 69

INSTITUTE OF MATHEMATICS

POLISH ACADEMY OF SCIENCES

WARSZAWA 2005

\title{
TRACELESS CUBIC FORMS ON STATISTICAL MANIFOLDS AND TCHEBYCHEV GEOMETRY
}

\author{
HIROSHI MATSUZOE \\ Department of Computer Science and Engineering \\ Graduate School of Engineering \\ Nagoya Institute of Technology \\ Gokiso-cho, Showa-ku, Nagoya, 466-8555, Japan \\ E-mail: matsuzoe@nitech.ac.jp
}

\begin{abstract}
Geometry of traceless cubic forms is studied. It is shown that the traceless part of the cubic form on a statistical manifold determines a conformal-projective equivalence class of statistical manifolds. This conformal-projective equivalence on statistical manifolds is a natural generalization of conformal equivalence on Riemannian manifolds. As an application, Tchebychev type immersions in centroaffine immersions of codimension two are studied.
\end{abstract}

1. Introduction. The purpose of this paper is to study geometry of traceless cubic forms on statistical manifolds. Cubic forms and their traceless parts have been studied in affine differential geometry. In particular, it is known that an affine hypersurface lies on a quadratic if its cubic form vanishes everywhere on the hypersurface (e.g. [1]).

In affine hypersurface theory, the traceless cubic form is invariant under choice of equiaffine transversal vector fields. (In other words, we often say that a traceless cubic form is invariant under relative normalizations.) We generalize this property, namely, we show that the traceless cubic form on a statistical manifold is invariant under conformalprojective changes of statistical manifolds. Moreover, we show that the traceless cubic form determines a conformal-projective class of statistical manifolds. The notion of conformal-projective class of statistical manifolds was introduced by the present author in [6]. This class induces conformal-projective geometry of statistical manifolds, which is a natural generalization of conformal geometry of Riemannian manifolds.

Traceless cubic forms are useful objects in Tchebychev hypersurface theory, and conformal-projective relations are useful objects in geometry of centroaffine immersions

2000 Mathematics Subject Classification: Primary 53A15; Secondary 53A30, 53B05, 53B25.

Key words and phrases: traceless cubic form, conformal-projective geometry, Tchebychev geometry, centroaffine immersion of codimension two, statistical manifold.

The paper is in final form and no version of it will be published elsewhere. 
of codimension two. Applying the conformal-projective geometry to Tchebychev hypersurface theory, we introduce Tchebychev type immersions in centroaffine immersions of codimension two.

We remark that several fundamental results in conformal-projective geometry have been obtained in Kurose's recent work [3]. Tchebychev geometry has interesting applications to Bayesian statistics. See the recent work by Takeuchi, Amari and the author [7].

2. Cubic forms on statistical manifolds. In this section, we summarize the fundamental facts about cubic forms.

Let $M$ be an $n$-dimensional manifold, let $h$ be a semi-Riemannian metric on $M$, and let $\nabla$ be a torsion-free affine connection on $M$. We say that the triplet $(M, \nabla, h)$ is a statistical manifold if $(\nabla, h)$ is a Codazzi pair on $M$, that is, $\nabla h$ is a totally symmetric (0,3)-tensor field on $M$.

We can define the dual connection (or conjugate connection) $\nabla^{*}$ of $\nabla$ with respect to $h$ by

$$
X h(Y, Z)=h\left(\nabla_{X} Y, Z\right)+h\left(Y, \nabla_{X}^{*} Z\right) .
$$

The connection $\nabla^{*}$ is torsion-free, and the covariant derivative $\nabla^{*} h$ is totally symmetric. Hence $\left(M, \nabla^{*}, h\right)$ is also a statistical manifold. We call $\left(M, \nabla^{*}, h\right)$ the dual statistical manifold of $(M, \nabla, h)$. Denote by $\nabla^{0}$ the Levi-Civita connection of $h$. Then $\nabla^{0}$ satisfies

$$
\nabla^{0}=\frac{1}{2}\left(\nabla+\nabla^{*}\right)
$$

We define tensor fields of type $(0,3)$ and of type $(1,2)$ by

$$
\begin{aligned}
C(X, Y, Z) & :=\left(\nabla_{X} h\right)(Y, Z), \\
K(X, Y) & :=K_{X} Y:=\frac{1}{2}\left(\nabla_{X} Y-\nabla_{X}^{*} Y\right) .
\end{aligned}
$$

From the definition of statistical manifolds, $C$ is totally symmetric. Since $\nabla$ and $\nabla^{*}$ are torsion-free, $K$ is also symmetric. Moreover, $C$ and $K$ satisfy the following formulas:

Proposition 2.1.

$$
\begin{aligned}
C(X, Y, Z) & =-2 h\left(K_{X} Y, Z\right), \\
K_{X} Y & =\nabla_{X} Y-\nabla_{X}^{0} Y=\nabla_{X}^{0} Y-\nabla_{X}^{*} Y .
\end{aligned}
$$

For proof, see Chapter 2 in [8], for example.

Proposition 2.2. Denote by $R$ and $R^{*}$ the curvature tensors of $\nabla$ and $\nabla^{*}$, respectively. Then

$$
\begin{aligned}
\left(\nabla_{X}^{0} C\right)(Y, Z, V)-\left(\nabla_{Y}^{0} C\right)(X, Z, V) & =h\left(R^{*}(X, Y) Z-R(X, Y) Z, V\right), \\
\left(\nabla_{X}^{0} K\right)(Y, Z)-\left(\nabla_{Y}^{0} K\right)(X, Z) & =\frac{1}{2}\left(R(X, Y) Z-R^{*}(X, Y) Z\right) .
\end{aligned}
$$

Proof. It is straightforward to show the formulas. See also Section 4.4.10 in [10], for example.

The tensor fields $C$ and $K$ are called the cubic form on $(M, \nabla, h)$. Sometimes $K$ is also called the difference tensor field [8] since $K$ represents the difference of two affine connections. 
We define a 1 -form $T^{b}$ by the trace of cubic form $K$ :

$$
n T^{b}(X):=\operatorname{tr}\left\{Y \mapsto K_{X} Y\right\} \quad\left(=-\frac{1}{2} \operatorname{tr}_{h}\{(Y, Z) \mapsto C(X, Y, Z)\}\right) .
$$

The 1 -form $T^{b}$ is called the Tchebychev form on a statistical manifold $(M, \nabla, h)$. Denote by $T$ the metrical dual vector field of $T^{b}$, namely, $h(X, T)=T^{b}(X)$. The vector field $T$ is called the Tchebychev vector field. For a statistical manifold $(M, \nabla, h)$, since $\nabla$ is assumed to be torsion-free, the Tchebychev form $T^{b}$ is exact if and only if the Ricci tensor of $\nabla$ is symmetric (see Section 4.4 .8 in [10]).

We define the traceless cubic form (or the traceless difference tensor $\widetilde{K}$ on a statistical manifold $(M, \nabla, h)$ by

$$
\widetilde{K}_{X} Y=K_{X} Y-\frac{n}{n+2}\left(h(X, Y) T+T^{b}(Y) X+T^{b}(X) Y\right) .
$$

Contracting the above equation, we can easily check $\operatorname{tr}\left\{Y \mapsto \tilde{K}_{X} Y\right\}=0$.

3. Conformal-projective changes of statistical manifolds. In this section, we recall conformal-projective geometry of statistical manifolds.

Suppose that $(M, \nabla, h)$ and $(M, \bar{\nabla}, \bar{h})$ are statistical manifolds. We say that $(M, \nabla, h)$ and $(M, \bar{\nabla}, \bar{h})$ are conformally-projectively equivalent if there exist two functions $\psi$ and $\phi$ on $M$ such that

$$
\begin{aligned}
\bar{h}(X, Y) & =e^{\psi+\phi} h(X, Y), \\
\bar{\nabla}_{X} Y & =\nabla_{X} Y-h(X, Y) \operatorname{grad}_{h} \psi+d \phi(Y) X+d \phi(X) Y,
\end{aligned}
$$

and the change $(\nabla, h) \mapsto(\bar{\nabla}, \bar{h})$ is called a conformal-projective change.

A statistical manifold $(M, \nabla, h)$ is said to be conformally-projectively flat if $(M, \nabla, h)$ is locally conformally-projectively equivalent to some flat statistical manifold. Here a statistical manifold $(M, \nabla, h)$ is said to be flat if the connection $\nabla$ is flat.

Suppose that $(M, g)$ and $(M, \bar{g})$ are Riemannian manifolds. If $\bar{g}=e^{2 \phi} g$ for some function $\phi$, then their Levi-Civita connections $\bar{\nabla}^{0}$ and $\nabla^{0}$ satisfy

$$
\bar{\nabla}_{X}^{0} Y=\nabla_{X}^{0} Y-g(X, Y) \operatorname{grad}_{g} \phi+d \phi(Y) X+d \phi(X) Y .
$$

Hence the conformal-projective equivalence on statistical manifolds is a generalization of a conformal equivalence on Riemannian manifolds.

This conformal-projective equivalence relation includes further equivalence relations on statistical manifolds. If the function $\psi$ in (3) and (4) is constant, then the connections $\nabla$ and $\bar{\nabla}$ are projectively equivalent. In this case, $(M, \nabla, h)$ and $(M, \bar{\nabla}, \bar{h})$ are said to be $(-1)$-conformally equivalent. If the function $\phi$ is constant, then the connections are dual-projectively equivalent [2], which is a well-known relation in affine hypersurface theory. In this case, $(M, \nabla, h)$ and $(M, \bar{\nabla}, \bar{h})$ are said to be 1-conformally equivalent.

The dual statistical manifolds have the following formula.

Proposition 3.1. Suppose that two statistical manifolds $(M, \nabla, h)$ and $(M, \bar{\nabla}, \bar{h})$ are conformally-projectively equivalent and satisfy the formulas (3) and (4). Then their dual 
statistical manifolds $\left(M, \nabla^{*}, h\right)$ and $\left(M, \bar{\nabla}^{*}, \bar{h}\right)$ are also conformally-projectively equivalent and the dual connections satisfy

$$
\bar{\nabla}_{X}^{*} Y=\nabla_{X}^{*} Y-h(X, Y) \operatorname{grad}_{h} \phi+d \psi(Y) X+d \psi(X) Y .
$$

Proof. We can obtain the result from straightforward calculations. See also Section 2 in [6] or Section 2 in [3].

For a statistical manifold $(M, \nabla, h)$, we denote by $R$ the curvature tensor of $\nabla$, by Ric and Ric* the Ricci tensors of $\nabla$ and $\nabla^{*}$, respectively. The Ricci operator Ric ${ }^{\#}$ is determined by $h\left(\operatorname{Ric}^{\#}(X), Y\right):=\operatorname{Ric}(X, Y)$, and the scalar curvature $\sigma$ is determined by the trace of the Ricci operator, namely, $\sigma:=\operatorname{tr} \operatorname{Ric}^{\#}=\operatorname{tr}_{h}$ Ric.

We define the conformal-projective curvature tensor $W$ of $(M, \nabla, h)$ by

$$
\begin{aligned}
W(X, Y) Z=R & (X, Y) Z \\
& -\{h(Y, Z) A(X)-h(X, Z) A(Y)+B(Y, Z) X-B(X, Z) Y\} \\
& -\frac{\sigma}{n(n-1)}\{h(Y, Z) X-h(X, Z) Y\},
\end{aligned}
$$

where $A$ and $B$ are the tensor fields of type $(1,1)$ and of type $(0,2)$, respectively, given by

$$
\begin{aligned}
A(X) & :=\frac{1}{n(n-2)}\left\{\operatorname{Ric}^{\#}(X)+(n-1) \operatorname{Ric}^{* \#}(X)-\sigma X\right\}, \\
B(X, Y) & :=\frac{1}{n(n-2)}\left\{(n-1) \operatorname{Ric}(X, Y)+\operatorname{Ric}^{*}(X, Y)-\sigma h(X, Y)\right\} .
\end{aligned}
$$

The conformal-projective curvature tensor is a generalization of Weyl's conformal curvature tensor in Riemannian geometry. Indeed, the following results hold.

Proposition 3.2. Let $(M, \nabla, h)$ and $(M, \bar{\nabla}, \bar{h})$ be statistical manifolds of dimension $n \geq 2$. If they are conformally-projectively equivalent, then their conformal-projective curvature tensors coincide.

Proof. See Section 4 in [3].

TheOREm $3.3([3])$. Let $(M, \nabla, h)$ be a statistical manifold of dimension $n \geq 4$. Then it is conformally-projectively flat if and only if the conformal-projective curvature tensor $W$ vanishes everywhere on $M$.

Proof. See Theorem 1.2 in [3].

4. Traceless cubic forms and conformal-projective geometry. In this section, we consider traceless cubic forms on statistical manifolds in terms of conformal-projective geometry.

Proposition 4.1. Suppose that two statistical manifolds $(M, \nabla, h)$ and $(M, \bar{\nabla}, \bar{h})$ are conformally-projectively equivalent. Then their traceless cubic forms $\tilde{K}$ and $\widetilde{\bar{K}}$ coincide.

Proof. Since $(M, \nabla, h)$ and $(M, \bar{\nabla}, \bar{h})$ are conformally-projectively equivalent, then $\nabla$ and $\bar{\nabla}$ satisfy (4) and their dual connections satisfy (5). Hence the cubic form $\bar{K}$ on $(M, \bar{\nabla}, \bar{h})$ is given by

(6) $2 \bar{K}_{X} Y=2 K_{X} Y-h(X, Y)\left(\operatorname{grad}_{h} \psi-\operatorname{grad}_{h} \phi\right)-(d \psi-d \phi)(Y) X-(d \psi-d \phi)(X) Y$. 
Contracting (6), we have

$$
\bar{T}^{b}(X)-T^{b}(X)=-\frac{n+2}{2 n}(d \psi-d \phi)(X) .
$$

Substituting (7) into (6), we can see $\tilde{K}=\widetilde{\bar{K}}$.

Conversely, a traceless cubic form determines a class of conformal-projective structure on a statistical manifold. This is our main theorem.

THEOREM 4.2. Let $(M, \nabla, h)$ and $(M, \bar{\nabla}, \bar{h})$ be simply connected statistical manifolds. Suppose that the Ricci tensor of $\nabla$ is symmetric and the Ricci tensor of $\bar{\nabla}$ is also symmetric. Suppose that $h$ and $\bar{h}$ are conformally equivalent, and the traceless cubic forms $\tilde{K}$ and $\widetilde{\bar{K}}$ coincide. Then $(M, \nabla, h)$ and $(M, \bar{\nabla}, \bar{h})$ are conformally-projectively equivalent.

Proof. Since $h$ and $\bar{h}$ are conformally equivalent, there exists a function $\phi_{1}$ such that $\bar{h}(X, Y)=e^{\phi_{1}} h(X, Y)$. Then their Levi-Civita connections satisfy

$$
\bar{\nabla}_{X}^{0} Y=\nabla_{X}^{0} Y-\frac{1}{2} h(X, Y) \operatorname{grad}_{h} \phi_{1}+\frac{1}{2}\left(d \phi_{1}(Y) X+d \phi_{1}(X) Y\right) .
$$

Denote by $T^{b}$ and $\bar{T}^{b}$ the Tchebychev forms on $(M, \nabla, h)$ and $(M, \bar{\nabla}, \bar{h})$, respectively. Since the Ricci tensors are symmetric and $M$ is simply connected, there exist functions $\psi_{0}$ and $\bar{\psi}_{0}$ satisfying $T^{b}=d \psi_{0}$ and $\bar{T}^{b}=d \bar{\psi}_{0}$.

Set $\psi_{1}=\psi_{0}-\bar{\psi}_{0}$. From the assumption $\widetilde{\bar{K}}=\tilde{K}$, straightforward calculation shows that

$$
\bar{K}_{X} Y=K_{X} Y-\frac{n}{n+2}\left(h(X, Y) \operatorname{grad}_{h} \psi_{1}+d \psi_{1}(Y) X+d \psi_{1}(X) Y\right) .
$$

Set $\psi=\frac{1}{2} \phi_{1}+\frac{n}{n+2} \psi_{1}, \phi=\frac{1}{2} \phi_{1}-\frac{n}{n+2} \psi_{1}$. Substituting (8) to (9), we have

$$
\begin{aligned}
\bar{h}(X, Y) & =e^{\psi+\phi} h(X, Y), \\
\bar{\nabla}_{X} Y & =\nabla_{X} Y-h(X, Y) \operatorname{grad}_{h} \psi+d \phi(Y) X+d \phi(X) Y .
\end{aligned}
$$

This implies that two statistical manifolds $(M, \nabla, h)$ and $(M, \bar{\nabla}, \bar{h})$ are conformallyprojectively equivalent.

\section{Traceless cubic forms and centroaffine immersions of codimension two.} In this section, we consider geometry of traceless cubic forms in terms of centroaffine immersions of codimension two. For the details of centroaffine immersions of codimension two, see $[9]$.

Let $M$ be an $n$-dimensional manifold ( $n \geq 2$ ), let $f$ be an immersion from $M$ to $\boldsymbol{R}^{n+2}$, and let $\xi$ be a local vector field along $f$. We call a pair $\{f, \xi\}$ a centroaffine immersion of codimension two if, for each point $p \in M$, the tangent space $T_{f(p)} M$ is decomposed as

$$
T_{f(p)} \boldsymbol{R}^{n+2}=f_{*}\left(T_{p} M\right) \oplus \boldsymbol{R} \xi_{p} \oplus \boldsymbol{R} f(p),
$$

where $\boldsymbol{R} \xi_{p}$ and $\boldsymbol{R} f(p)$ are 1-dimensional subspaces spanned by $\xi_{p}$ and $f(p)$, respectively. Identifying $T_{f(p)} \boldsymbol{R}^{n+2}$ as $\boldsymbol{R}^{n+2}$, the above equation implies that $\xi_{p}$ and $f(p)$ are transversal to $f(M)$. We call $\xi$ a transversal vector field. 
Denote by $D$ the standard flat affine connection of $\boldsymbol{R}^{n+2}$. The covariant derivative is accordingly decomposed as follows:

$$
\begin{aligned}
D_{X} f_{*} Y & =f_{*}\left(\nabla_{X} Y\right)+h(X, Y) \xi+k(X, Y) f, \\
D_{X} \xi & =-f_{*}(S X)+\tau(X) \xi+\mu(X) f .
\end{aligned}
$$

We call $\nabla$ the induced connection, $h$ the affine fundamental form, $\tau$ the transversal connection form and $S$ the affine shape operator.

For a centroaffine immersion of codimension two, the following structural equations hold:

Gauss:

$$
R(X, Y) Z=h(Y, Z) S X-h(X, Z) S Y-k(Y, Z) X+k(X, Z) Y,
$$

Codazzi:

$$
\begin{aligned}
& \left(\nabla_{X} k\right)(Y, Z)+\mu(X) h(Y, Z)=\left(\nabla_{Y} k\right)(X, Z)+\mu(Y) h(X, Z), \\
& \left(\nabla_{X} h\right)(Y, Z)+\tau(X) h(Y, Z)=\left(\nabla_{Y} h\right)(X, Z)+\tau(Y) h(X, Z), \\
& \left(\nabla_{X} S\right)(Y)-\tau(X) S Y+\mu(X) Y=\left(\nabla_{Y} S\right)(X)-\tau(Y) S X+\mu(Y) X,
\end{aligned}
$$

Ricci:

$$
\begin{aligned}
& k(X, S Y)-k(Y, S X)=\left(\nabla_{X} \mu\right)(Y)-\left(\nabla_{Y} \mu\right)(X)+\tau(Y) \mu(X)-\tau(X) \mu(Y), \\
& h(X, S Y)-h(Y, S X)=\left(\nabla_{X} \tau\right)(Y)-\left(\nabla_{Y} \tau\right)(X) .
\end{aligned}
$$

Contracting Gauss equation, we have

$$
\operatorname{Ric}(Y, Z)=\operatorname{tr} S h(Y, Z)-h(S Y, Z)-(n-1) k(Y, Z) .
$$

Now we check how various induced quantities depend on $\xi$. Set

$$
\xi^{\prime}=e^{-\psi}\left(\xi+a f+f_{*} U\right) .
$$

Then the induced quantities $\nabla, h$ and $\tau$ change as follows:

$$
\begin{aligned}
\nabla_{X}^{\prime} Y & =\nabla_{X} Y-h(X, Y) U, \\
h^{\prime}(X, Y) & =e^{\psi} h(X, Y), \\
\tau^{\prime}(X) & =\tau(X)-d \psi(X)+h(X, U) .
\end{aligned}
$$

Two centroaffine immersions $f$ and $\bar{f}$ are said to be projectively equivalent if $\bar{f}=e^{\phi} f$ for some function $\phi$, and we call the change $f \mapsto \bar{f}$ a projective change. For a projective change $\bar{f}=e^{\phi} f$, induced quantities $\bar{\nabla}, \bar{h}$ and $\bar{\tau}$ of $\{\bar{f}, \xi\}$ are given as follows:

$$
\begin{aligned}
\bar{\nabla}_{X} Y & =\nabla_{X} Y+d \phi(Y) X+d \phi(X) Y, \\
\bar{h}(X, Y) & =e^{\phi} h(X, Y), \\
\bar{\tau} & =\tau .
\end{aligned}
$$

We say that $f$ is non-degenerate if $h$ is non-degenerate. From Equation (16), the definition is independent of choice of $\xi$. We say that $\{f, \xi\}$ is equiaffine if $\tau=0$. If $\{f, \xi\}$ is non-degenerate and equiaffine, from Codazzi equation (11), the triplet of induced objects $(M, \nabla, h)$ is a statistical manifold. 
If we exchange transversal vector fields equiaffinely, then the tangent vector $U$ in (14) is given by the gradient vector of $\psi$ from Equation (17):

$$
U=\operatorname{grad}_{h} \psi \text {. }
$$

Hence we obtain a conformal-projective equivalence relation from a change of transversal vector fields and a projective change.

Proposition 5.1 ([6]). Let $\{f, \xi\}$ be a non-degenerate equiaffine centroaffine immersion of codimension two. Denote by $\nabla$ the induced connection, and by $h$ the affine fundamental form of $\{f, \xi\}$. Then $(M, \nabla, h)$ is a conformally-projectively flat statistical manifold.

Suppose that $\{f, \xi\}$ is non-degenerate and equiaffine. Denote by $\boldsymbol{R}_{n+2}$ the dual space of $\boldsymbol{R}^{n+2}$. Then the dual map $\{v, w\}$ of $\{f, \xi\}$ is defined by

$$
\begin{gathered}
\left\langle v(p), \xi_{p}\right\rangle=1,\langle v(p), f(p)\rangle=0,\left\langle v(p), f_{*} X_{p}\right\rangle=0, \\
\left\langle w(p), \xi_{p}\right\rangle=0,\langle w(p), f(p)\rangle=1,\left\langle w(p), f_{*} X_{p}\right\rangle=0,
\end{gathered}
$$

where $\langle$,$\rangle is the pairing of \boldsymbol{R}_{n+2}$ and $\boldsymbol{R}^{n+2}$. The dual map $\{v, w\}$ is a centroaffine immersion of codimension two from $M$ to $\boldsymbol{R}_{n+2}$ since $h$ is non-degenerate. We denote by $\nabla^{*}, h^{*}, S^{*}, \ldots$ the induced objects of $\{v, w\}$. Then we have the following formulas:

$$
\begin{aligned}
X h(Y, Z) & =h\left(\nabla_{X} Y, Z\right)+h\left(Y, \nabla_{X}^{*} Z\right), \\
h^{*}(X, Y) & =h(X, Y), \\
k^{*}(X, Y) & =-h(S X, Y), \\
h\left(S^{*} X, Y\right) & =-k(X, Y), \\
\tau^{*} & =0 .
\end{aligned}
$$

Now we consider Tchebychev type immersions in centroaffine immersions of codimension two. The following proposition is a direct analogue of the result in affine hypersurface theory.

Proposition $5.2([5])$. Let $\{f, \xi\}$ be a non-degenerate equiaffine centroaffine immersion of codimension two. Denote by $\nabla^{0}$ the Levi-Civita connection of the affine fundamental form $h$, by $T$ the Tchebychev vector field, and by $\tilde{K}$ the traceless cubic form of $\{f, \xi\}$. Then the following conditions are equivalent:

(1) $\left(\nabla_{X}^{0} \tilde{K}\right)(Y, Z)=\left(\nabla_{Y}^{0} \tilde{K}\right)(X, Z)$.

(2) $L=\lambda$ id for some function $\lambda$, where $L$ is determined by $L:=\frac{1}{2} S-\frac{1}{2} S^{*}-\frac{n}{n+2} \nabla^{0} T$.

(3) $\operatorname{div} \tilde{K}=0$.

Proof. The proof is also an analogue of Proposition 2.3 in [5]. From Gauss equation (10) and Equation (25), the curvature tensor $R$ satisfies

$$
R(X, Y) Z=h(Y, Z) S X-h(X, Z) S Y+h\left(S^{*} Y, Z\right) X-h\left(S^{*} X, Z\right) Y .
$$

From Ricci equations (12) and (13), the Ricci tensor of $\nabla$ is symmetric since $\xi$ is equiaffine. This implies that the Tchebychev form $T^{b}$ is exact. 
Form the definition of traceless cubic form, Proposition 2.2, and Equation (26), using $d T^{b}=0$, we have

$$
\begin{aligned}
\left(\nabla_{X}^{0} \tilde{K}\right)(Y, Z)-\left(\nabla_{Y}^{0} \tilde{K}\right)(X, Z) & \\
& =h(Y, Z) L X-h(X, Z) L Y+h(L X, Z) Y-h(L Y, Z) X .
\end{aligned}
$$

$(2) \Rightarrow(1)$ : This is trivial from (27).

$(1) \Rightarrow(2)$ : From the assumption, we have

$$
h(Y, Z) L X-h(X, Z) L Y+h(L X, Z) Y-h(L Y, Z) X=0 .
$$

Contracting the equation above, we have

$$
h(Y, Z) \operatorname{tr} L-h(L Y X, Z)+h(L Y, Z)-n h(L Y, Z)=0 .
$$

This implies that $L Y=\frac{1}{n} \operatorname{tr} L Y$.

$(2) \Leftrightarrow(3)$ : This is quite same as in [5].

We say that a non-degenerate equiaffine immersion $\{f, \xi\}$ is of Tchebychev type if $L=$ $\lambda i d$ for some function $\lambda$. This definition is independent of choice of equiaffine transversal vector field $\xi$ and projective changes of $f$ from Equations (15)-(21) and Theorem 4.2.

Tchebychev type immersions in centroaffine immersions of codimension two naturally generalize Tchebychev hypersurface immersions or Tchebychev type immersions into a space form of constant curvature. For example, the following proposition generalizes the result on Tchebychev type immersions into a space form of constant curvature [4].

Proposition 5.3. Suppose that $\{f, \xi\}$ is a non-degenerate equiaffine centroaffine immersion of codimension two. Then $\{f, \xi\}$ is Tchebychev type if and only if its dual map $\{v, w\}$ is also Tchebychev type.

Proof. $L^{*}=\frac{1}{2} S^{*}-\frac{1}{2} S+\frac{n}{n+2} \nabla^{0} T=-L$.

The following theorem is a characterization of Tchebychev type immersions.

THEOREM 5.4. Suppose that $\{f, \xi\}$ is a Tchebychev type immersion. Then there exists a Tchebychev hypersurface immersion $\{\bar{f}, \bar{\xi}\}$ to $\boldsymbol{R}^{n+1} \subset \boldsymbol{R}^{n+2}$, at least locally, such that $f$ is projectively equivalent to $\bar{f}$.

Proof. Since $\{f, \xi\}$ is a centroaffine immersion, we can change the immersion $\bar{f}=e^{\phi} f$ for some function $\phi$, at least locally, such that $\bar{f}$ is contained in an affine hyperplane which does not pass through the origin. We denote the hyperplane by $\boldsymbol{R}^{n+1}$.

We can take a transversal vector field $\bar{\xi}$ which is contained in the hyperplane $\boldsymbol{R}^{n+1}$. This implies that $\{\bar{f}, \bar{\xi}\}$ is an affine hypersurface immersion. Denote by $\bar{\nabla}, \bar{h}, \bar{S}, \ldots$ the induced objects of $\{\bar{f}, \bar{\xi}\}$. We accordingly have $\bar{k}=0$, and $\bar{S}^{*}=0$.

Since the traceless cubic form is independent of projective changes and choice of equiaffine transversal vector fields, we have

$$
\bar{L}=\frac{1}{2} \bar{S}-\frac{n}{n+2} \bar{\nabla}^{0} \bar{T}=\lambda i d,
$$

for some function $\lambda$. This implies that $\bar{f}$ is a Tchebychev hypersurface immersion (see $[5]$ ). From the definition of $\bar{f}$, the Tchebychev type immersion $f$ is projectively equivalent to $\bar{f}$. 
Recall that $\tilde{K}=0$ characterized hyperquadratic in affine hypersurface theory. Hence we have the following corollary. (This corollary was obtained in [9] in a more general setting.)

COROLlARY 5.5. If a non-degenerate equiaffine centroaffine immersion of codimension two $\{f, \xi\}$ satisfies that $\tilde{K}=0$, then the image lies on a quadratic cone.

Acknowledgements. This research was carried out while the author stayed at Technische Universität Berlin. The author would like to express his gratitude to Professor Udo Simon for his kind hospitality. In addition, the author would like to express his gratitude to the referee for valuable comments on this paper.

\section{References}

[1] F. Dillen, G. Verbouwe and L. Vrancken, Cubic form geometry for immersions in centroaffine and graph hypersurfaces, Results Math. 43 (2003), 88-95.

[2] S. Ivanov, On dual-projectively flat affine connections, J. Geom. 53 (1995), 89-99.

[3] T. Kurose, Conformal-projective geometry of statistical manifolds, Interdiscip. Inform. Sci. 8(2002), 89-100.

[4] T. Lusala, Tchebychev hypersurfaces of $S^{n+1}(1)$, in: Geometry and Topology of Submanifolds X, World Sci., 2000, 154-170.

[5] A.-M. Li, H. Liu, A. Schwenk-Schellschmidt, U. Simon and C. P. Wang, Cubic form methods and relative Tchebychev hypersurfaces, Geom. Dedicata 66 (1997), 203-221.

[6] H. Matsuzoe, On realization of conformally-projectively flat statistical manifolds and the divergences, Hokkaido Math. J. 27 (1998), 409-421.

[7] H. Matsuzoe, J. Takeuchi and S. Amari, Equiaffine structures on statistical manifolds and Bayesian statistics, to appear in Diff. Geom. Appl.

[8] K. Nomizu and T. Sasaki, Affine Differential Geometry-Geometry of Affine Immersions, Cambridge University Press, 1994.

[9] K. Nomizu and T. Sasaki, Centroaffine immersions of codimension two and projective hypersurface theory, Nagoya Math. J. 132 (1993), 63-90.

[10] U. Simon, A. Schwenk-Schellschmidt and H. Viesel, Introduction to the Affine Differential Geometry of Hypersurfaces, Lecture notes of the Science University of Tokyo, 1991. 